\title{
Laminar Turbulent Transition in a Boundary Layer Subjected to Weak Free Stream Turbulence*
}

\author{
Toshiaki KENCHI ${ }^{* *}$, Masaharu MATSUBARA** and Toshihiko IKEDA*** \\ ** Department of Mechanical Systems Engineering, Shinshu University \\ 4-17-1 Wakasato, Nagano-shi, Nagano 380-8553, Japan \\ *** Department of Environmental Science and Technology, Shinshu University \\ 4-17-1 Wakasato, Nagano-shi, Nagano 380-8553, Japan
}

\begin{abstract}
For revealing the transition process in a flat plate boundary layer subjected to a weak free stream turbulence, flow visualization and hot-wire measurements were performed. A weak free stream turbulence was generated by a turbulence grid mounted upstream of the contraction. The flow visualization clearly displayed a transition scenario in which a local two-dimensional wave packet rapidly forms a $\Lambda$ shape structure and then breaks down to turbulence, resulting in the generation of a turbulent spot. Quantitative measurements performed by using a hot-wire anemometer also confirmed the existence of local Tollmien-Schlichting waves that agreed with the parallel linear theory in terms of their frequency, phase velocity, and the wall-normal distribution of band-pass-filtered fluctuations. For comparison, a boundary layer subjected to a moderate-intensity free stream turbulence was investigated. This investigation showed that streaky structures play an important role in the boundary layer transition, as shown by Matsubara et al. [ J. Fluid Mech., 430, (2001), 149-168. ] A drastic change occurred in the transition process and this change could be sensitively determined by employing the intensity and/or spectra of the free stream turbulence.
\end{abstract}

Key words : Transition, Stability, Boundary Layer, Free Stream Turbulence, TollmienSchlichting Wave, Wave Packet

\section{Introduction}

The need for the establishment of a prediction method for predicting laminar-turbulent transition has been felt for a long time in most engineering fields related to fluid dynamics. It is not an exaggeration to state that the most general disturbance inducing a laminar-turbulent transition is the free stream turbulence; moreover, researches on the transition scenario in a boundary layer subjected to a free stream turbulence are limited. Early experiments on natural transition in a boundary layer subjected to considerably low free stream turbulences were performed by Schubauer and Skramstad ${ }^{(1)}$. They first confirmed the existence of TollmienSchlichting (T-S) waves by comparing their oscillations to the linear theory. The first intensive research by Klebanoff ${ }^{(2)}$ can unfortunately be referred to only indirectly through Kendall' $s^{(3)}$ report. Klebanoff mounted a turbulence grid that was constructed by using a rope in a settling chamber to obtain turbulence levels of as much as $0.3 \%$. While, he detected very low frequency disturbance with spanwise wavenumber, the so-called Klebanoff mode, he could obtain only indirect evidence of the emergence of T-S waves; however, in an artificially destabilized case, he could obtain direct evidence of the waves by using two-dimensional roughness. Kendall ${ }^{(4)}$ confirmed the presence of locally generated T-S waves in the flow visualizations and hot-wire signals for a free stream turbulence of a few percent, which was generated by a turbulence grid mounted in a settling chamber; it was subsequently concluded that envelopes of the T-S wave do not agree with the linear theory and depend on the presence of 


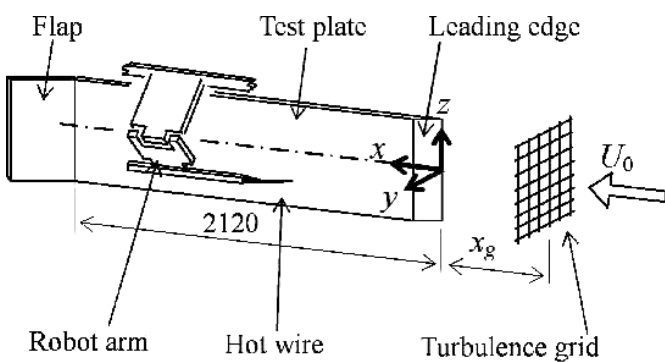

Fig. 1 Experimental setup. (dimensions in millimeters)

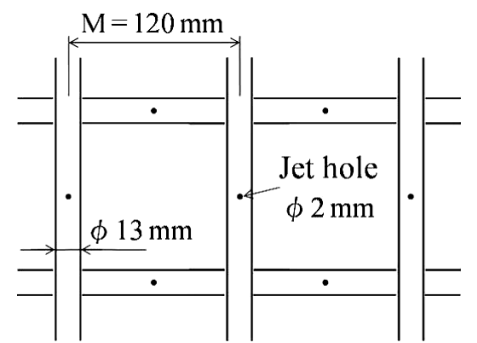

Fig. 2 Schematic view of turbulence grid A.

Table 1 Details of turbulence grids.

\begin{tabular}{cccccc}
\hline grid & streamwise position & mesh size & bar geometry & bar width & solidity \\
of grid $x_{g}$ & $\mathrm{M}$ & & & \\
A & $-3800 \mathrm{~mm}$ & $120 \mathrm{~mm}$ & circular & $13 \mathrm{~mm}$ & $20 \%$ \\
B & $-1410 \mathrm{~mm}$ & $50 \mathrm{~mm}$ & square & $6 \mathrm{~mm}$ & $23 \%$ \\
\hline
\end{tabular}

nonlinearities in the free stream turbulence level. The streamwise position where the local T-S wave emerged and the frequency of the wave corresponded to a position downstream of the maximum growth position predicted by the linear theory for a parallel flow assumption. $\mathrm{He}$ also observed an increase in the number of cycles of the T-S wave downstream and a lower propagation speed in the spanwise direction as compared with Gaster' $\mathrm{s}^{(5)}$ results for locally generated T-S waves. Arnal et al. ${ }^{(6)}$ and Kosorygin et al. ${ }^{(7)}$ also observed disturbances whose frequency matched the theoretically predicted frequency for the T-S waves; however, both these studies could not confirm a T-S breakdown.

In the case of high free stream turbulence, above $1 \%$, streaky structures so-called nonmodal growth ${ }^{(8)}$ disturbance or Klebanoff mode oscillation play an important role in transitions. They grow even upstream of the critical Reynolds number and break down to turbulence with a spanwise wavy motion; subsequently, they generate turbulent spots ${ }^{(9)}$. Nonmodal growth disturbances are also observed in the case of a low free stream turbulence ${ }^{(4)}$; however, their role in laminar-turbulent transitions has not been clarified.

The aims of this paper are to confirm the presence of naturally generated T-S waves in a flat plate boundary layer subjected to a weak free stream turbulence and their breakdown to turbulence. First, flow visualization is performed for smoke injection quite close to a test wall, and subsequently single hot wire measurements are carried out.

\section{Experimental description}

A schematic view of the experimental setup is shown in Fig. 1. Experiments were performed in a closed wind tunnel, which contained a test chamber with a length, height, and width of $4 \mathrm{~m}, 0.6 \mathrm{~m}$, and $0.4 \mathrm{~m}$, respectively; the test chamber was preceded by a threedimensional contraction with a 9:1 ratio. One honeycomb and five screens were installed in a settling chamber for reducing the turbulence level of the free stream. A test plate that was $2120 \mathrm{~mm}$ long, $580 \mathrm{~mm}$ wide, and $10 \mathrm{~mm}$ thick was used and it had a 10:1 ellipse leading edge with a $100 \mathrm{~mm}$ major axis. The plate was mounted vertically in the test chamber. The leading edge was located $1250 \mathrm{~mm}$ downstream from the contraction exit.

The trailing-edge flap and tunnel wall opposite the test plate were carefully adjusted to prevent separation around the leading edge and to minimize the pressure gradient along the plate. The free stream turbulence level was $0.17 \%$ at a free stream velocity $U_{\infty}=10 \mathrm{~m} / \mathrm{s}$ and the spectra of the turbulence was concentrated in the low-frequency range less than $10 \mathrm{~Hz}$. The free stream velocity was defined above the plate $200 \mathrm{~mm}$ downstream from the leading edge. The turbulence intensity was $0.033 \%$ after low-pass filtering with a cutoff frequency of $13.8 \mathrm{~Hz}$, which corresponded to a wavelength equal to the cross-sectional diagonal of the test 


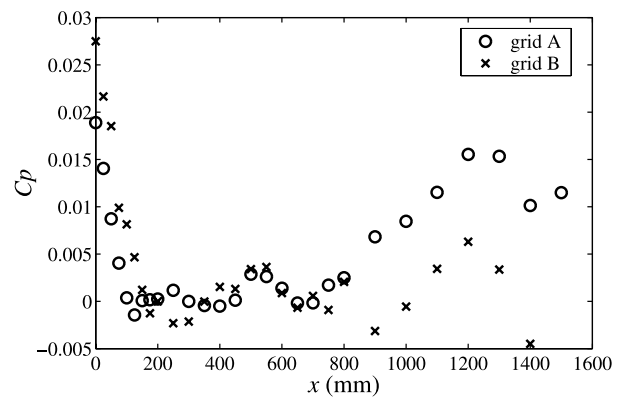

Fig. 3 Pressure distribution along the plate.

section.

In this experiment, two turbulence grids are used. Table 1 shows the details of the two grids. $x_{g}$ is the distance from the grid position to the leading edge. One is an active turbulence grid (Grid A) and is inserted $200 \mathrm{~mm}$ upstream of the contraction inlet to generate a weak free stream turbulence. The other is a passive turbulence grid (Grid B) that generates a high free stream turbulence and it is mounted downstream of the contraction. In the passive use of grid $\mathrm{A}$, the free stream turbulence is so weak that the transition is not completed within the measurable streamwise distance for $U_{\infty}=10 \mathrm{~m} / \mathrm{s}$. For increasing the free stream turbulence level, the pipes of grid A have holes with a diameter of $2 \mathrm{~mm}$ that are bored with an upstream orientation, as shown in Fig. 2. The air in the pipes is pressurized at $0.40 \mathrm{kPa}$ by a blower so that the jets blowing upstream from the holes increase the turbulence level of the free stream. This pressure level is maintained for the observation of the entire transition.

Experiments were performed with $U_{\infty}$ values of $12 \mathrm{~m} / \mathrm{s}$ and $6 \mathrm{~m} / \mathrm{s}$ for grid A and grid $\mathrm{B}$, respectively; these values corresponded to the unit Reynolds numbers $8 \times 10^{5} \mathrm{~m}^{-1}$ and $4 \times 10^{5} \mathrm{~m}^{-1}$, respectively. The coordinate system was set so that $x$ represents the streamwise direction; $y$, the wall-normal direction; and $z$, the direction normal to both the $x$ and $y$ directions. The origin of the $x$-axis was at the leading edge, and $y$ was measured from the surface of the test plate.

The velocity measurement was performed by using a constant temperature hot-wire anemometer that consisted of a $2.5 \mu$ m platinum wire with a length of $1.5 \mathrm{~mm}$. An X-probe was used for the free stream turbulence measurements and a single probe was employed for the boundary layer measurements. A three-axis robot arm, which was designed to minimize the flow resistance, traversed the hot-wire probe in the $y-z$ plane. Each joint of the arm was equipped with a stepping motor connected to harmonic gears whose backlash could not be measured. The position accuracy of the arm was less than $0.05 \mathrm{~mm}$ in the $y-z$ plane. For the streamwise movement, a rail with a length of $2250 \mathrm{~mm}$ was mounted over the test-section roof. The arm was driven by a motor comprising a rack-and-pinion gear.

Flow visualization was performed by making an alcohol mist carefully seep through a $2 \mathrm{~mm} \times 500 \mathrm{~mm}$ spanwise slot located at $x=210 \mathrm{~mm}$. Photographic recordings of the flow visualization were obtained by employing a six-megapixel camera positioned outside the transparent tunnel wall opposite the test plate. The shutter speed was $1 / 8000 \mathrm{~s}$. The smoke was illuminated by two flashlights through a window in the floor wall of the test section. A 0.31-megapixel digital video camera with a frame rate of $120 \mathrm{fps}$ and four $500 \mathrm{~W}$ floodlights were used for the video recordings. The placement of the lights and camera was the same as that for the photographic recording.

Figure 3 shows the streamwise pressure distribution in the free stream. The pressure coefficient is defined as $C_{p}=\left(U_{\infty}^{2}-U^{2}\right) / U_{\infty}^{2}$. In the case of both grids A and $\mathrm{B}$, the pressure coefficient is less than $0.5 \%$, except at $x<125 \mathrm{~mm}$ and $x>800 \mathrm{~mm}$. The large pressure exists because of the influence of the leading edge. Downstream of $x>800 \mathrm{~mm}$, the pressure coefficients slightly increase to a maximum of $1.5 \%$. For reference, in the setup of Klingmann 


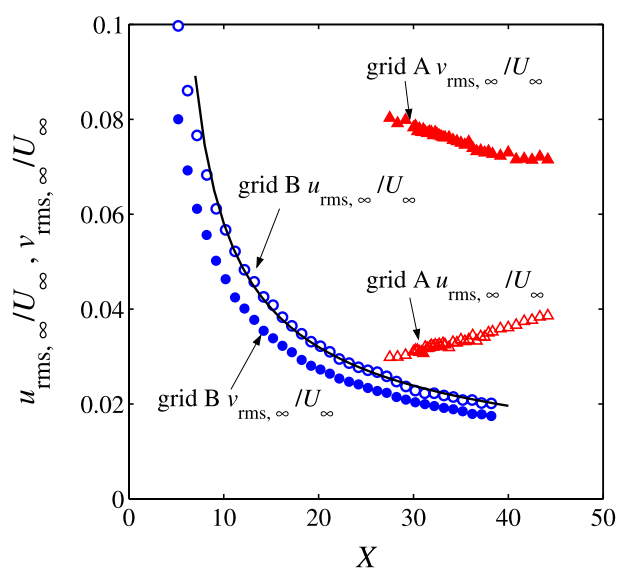

Fig. 4 Turbulence decay for grids A and B. The fluctuations of grid A are multiplied by 10 . The curve fitted to $u_{r m s, \infty} / U_{\infty}$ of grid $\mathrm{B}$ according to equation 1 only data for which $X \geq 10$ has been used.

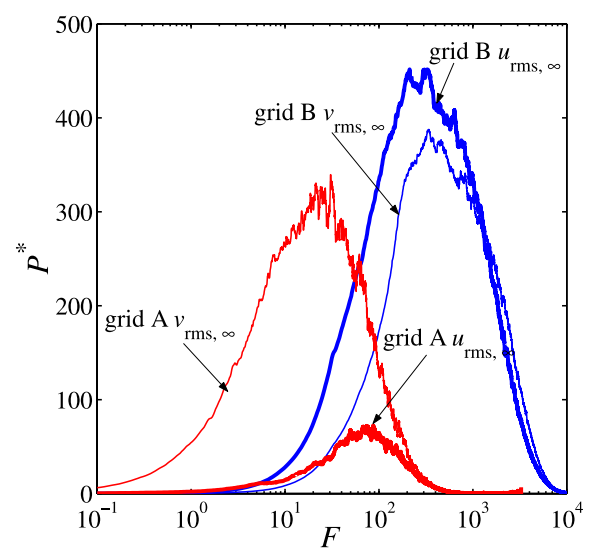

Fig. 5 free stream spectra at $x=0$. $P^{*}=F \times$ power. The power of grid A is multiplied by 50 .

et al. ${ }^{(10)}, C_{p}$ was constant to within $1 \%$, except near the leading edge of the plate. Kendall ${ }^{(4)}$ reported that $C_{p}$ was constant to within $0.07 \%$. Compared with the setups reported in other studies, the present one is sufficiently small. Additionally, the pressure gradient downstream of $x>800 \mathrm{~mm}$ does not affect the transition because the transition ends upstream of that area in the case of both grids $\mathrm{A}$ and $\mathrm{B}$.

\section{Decay of free stream turbulence and its spectra}

Figure 4 shows the downstream development of the streamwise velocity fluctuation $\left(u_{r m s, \infty}\right)$ and vertical velocity fluctuation $\left(v_{r m s, \infty}\right)$ in the free stream turbulence. The streamwise position is normalized by using the mesh size $\mathrm{M}$ according to the relation $X=\left(x-x_{g}\right) / \mathrm{M}$. A flow contraction region occurs for $4.58 \leq X \leq 21.25$ in the case of grid A.

In the case of grid $\mathrm{A}$, the vertical component immediately downstream of the contraction is $X=27.5$, which is 2.7 times greater than the streamwise component. Further downstream, the streamwise component increases downstream, while the wall-normal component decays. This redistribution of the directional energy component was previously observed by Uberoi ${ }^{(11)}$ by using a grid mounted upstream of a contraction. There exists an anisotropy $u_{r m s, \infty} / v_{r m s, \infty}=$ 0.42 at the streamwise position of the leading edge. Kendall ${ }^{(3)}$ also reported that $u_{r m s, \infty} / v_{r m s, \infty}$ for the free stream after contraction was in the range between 0.40 and 0.63 . The value of $v_{r m s, \infty}$ at the leading edge streamwise position is $0.77 \% U_{\infty}$ and $u_{r m s, \infty} / v_{r m s, \infty}=0.42$.

In the case of grid $\mathrm{B}$, both $u_{r m s, \infty}$ and $v_{r m s, \infty}$ decrease downstream while maintaining a ratio of approximately 1.15 independent of the streamwise position, except immediately downstream of the grid. This weak anisotropy is generally observed in grid turbulence ${ }^{(12)(13)}$. The curve in Fig. 4 indicates the typical power-law decay of grid turbulence according to Eq. 1:

$$
\frac{u_{r m s, \infty}}{U_{\infty}}=C\left(X-X_{0}\right)^{-b}
$$

where $X_{0}$ is the virtual origin of the grid and the constant $C$ and exponent $b$ are parameters to be determined by fitting a curve to the experimental data in the case of grid $\mathrm{B}$. The curve is in good agreement with the parameters $X_{0}=4.13, b=-0.60$, and $C=0.17$. The values of $b$ and the ratio $u_{r m s} / v_{r m s}$ are satisfactory as compared with those of other investigations on grid turbulence ${ }^{(12)}$. The streamwise component and vertical component above the leading edge at $X=28.2$ are $2.48 \% U_{\infty}$ and $2.16 \% U_{\infty}$. These values are considerably larger than the streamwise intensity in the case of grid A. The parameter $u_{r m s, \infty} / v_{r m s, \infty}$ is given by the value 1.15 , which shows that it is relatively small as compared with that in the case of grid A. 


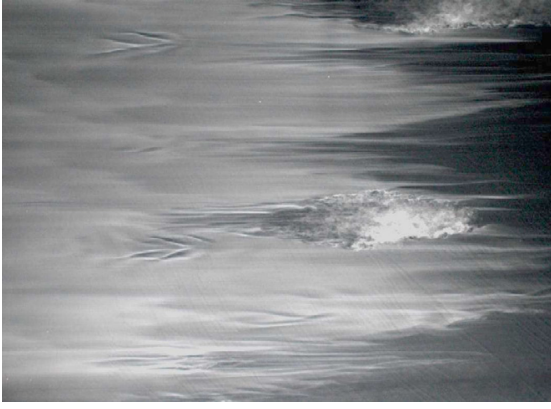

Fig. 6 The smoke visualization result in case of grid A. $2.64 \times 10^{5} \leq R e_{x} \leq 6.64 \times 10^{5}$.

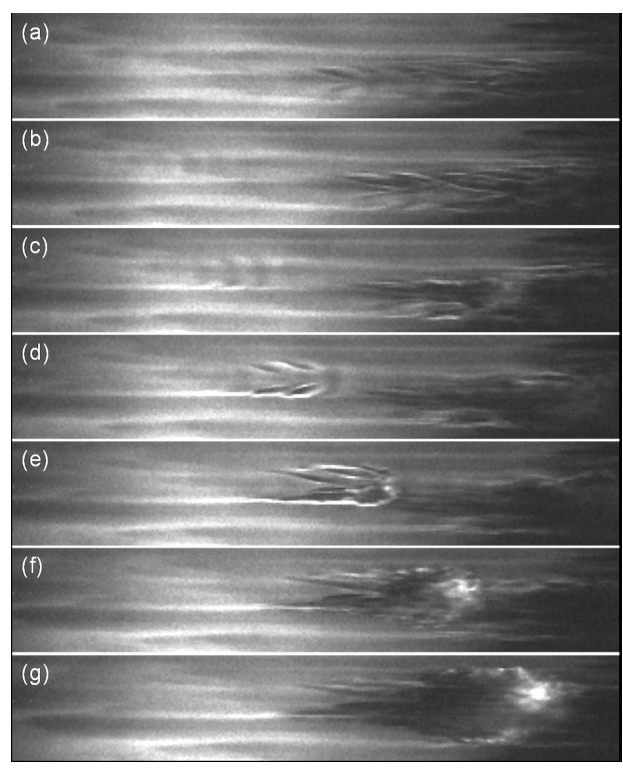

Fig. 7 Sequence of $\Lambda$ shape structure breakdowns. The interval between pictures is $8.3 \mathrm{~ms}$. Grid A. $2.88 \times 10^{5} \leq R e_{x} \leq 7.4 \times 10^{5}$.

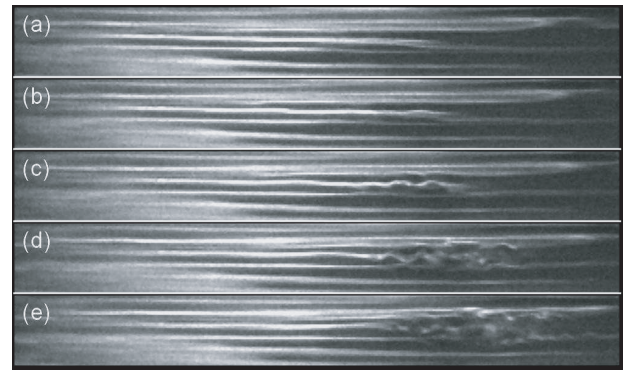

Fig. 9 Sequence of streak breakdowns. The interval between pictures is $8.3 \mathrm{~ms}$. Grid B. $1.2 \times 10^{5} \leq R e_{x} \leq 3.2 \times 10^{5}$.

Fig. 8 The smoke visualization result in case of grid B. $1.28 \times 10^{5} \leq R e_{x} \leq 3.48 \times 10^{5}$.

The spectral density distributions of each directional component for both grids A and $\mathrm{B}$ are compared in Fig. 5. The horizontal axis represents the normalized frequency $F=$ $2 \pi f v \times 10^{6} / U_{0}^{2}$, where $f$ is the frequency and $v$ is the kinematic viscosity. The vertical axis is premultiplied by $F$ so that the area enclosed by a spectral curve represents the energy of the free stream turbulence. In the case of grid A, the vertical component is higher than the streamwise component at low frequencies. However, the turbulence is nearly isotropic for frequencies in the typical T-S wave range (around $F=150$ ). Strong anisotropy in the lowfrequency range was also observed by Kendall ${ }^{(4)}$ in a free stream turbulence generated by a grid mounted upstream of a contraction. In the case of grid B, weak anisotropy is observed at low frequencies. These spectra and the decay behavior indicate a typical quasi-isotropic turbulence.

\section{Flow visualization}

Flow visualization is performed to investigate the disturbance structures and transition process in a boundary layer subjected to free stream turbulence. In the absence of the turbulence grids, the smoke forms a homogeneous layer and drifts on the surface of the test plate, indicating the laminar nature of the boundary layer. Figure 6 corresponds to the weak free stream turbulence case for grid A at $U_{\infty}=12 \mathrm{~m} / \mathrm{s}$. In the left-side region, the smoke is almost homogeneous and the light, thick, and thin patches indicate the existence of large-scale 


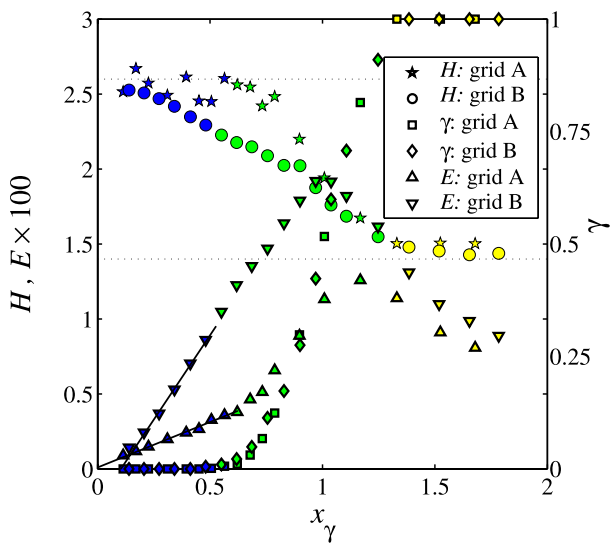

Fig. 10 Streamwise disturbance energy and intermittency as functions of $R e_{x}$ at $y / \delta^{*}=$ 1.2. The blue symbols corresponds to zero of intermittency. The green symbols indicate transitional zone. The yellow symbols corresponds to turbulence.

disturbance structures. In this quasi-homogeneous region, $\Lambda$ shape structures aligned in the streamwise direction are observed at the left-side center and upper part. The center structure is followed by local waves with a constant wavelength. The spanwise width of these $\Lambda$ shape structures is around four times the boundary layer thickness. In the visualization of Kendall ${ }^{(4)}$, periodic structures aligned in the streamwise direction are also observed, and their spanwise width is approximately three times the boundary layer thickness.

At the center and top of the right side of the image, there exist lumps of turbulence that are characterized by downstream-pointing V-shaped structures corresponding to a typical turbulent spot. From the video recordings, it is confirmed that the $\Lambda$ shape structures break down to form a turbulent spot, as shown in Fig. 7. On the left side of Fig. 7 (a), structures aligned in the streamwise direction are not observed. Feeble two-dimensional waves propagating in the streamwise direction appear in (b). In panel (c), that local two-dimensional waves with their wave fronts directed in the spanwise direction shapen. Subsequently in panel (d), they form $\Lambda$ shape structures, as observed in Fig. 6. In panels (e) to (g), the $\Lambda$ shape structures immediately break down and generate a turbulent spot. From video recordings over long durations, the number of the local two-dimensional waves is detected to be greater than 20 , and all these waves break down to turbulence along with the formation of $\Lambda$ shape structures.

Figure 8 shows a transitional boundary layer in the case of grid B. There exist strips that are extremely elongated in the streamwise direction with a spanwise scale comparable to the boundary layer thickness. These strips are recognized as streaky structures due to nonmodal growth $^{(9)}$. Turbulent spots are observed downstream. From video recordings, it is observed that the streak exhibits a wavy motion and subsequently breaks down to form a turbulent spot downstream, as shown in Fig. 9.

Comparing the two grid cases, the disturbances that play the main role in the transition scenario are observed to be completely different. In the case of grid B, the breakdown of the streaky structures triggers the transition to turbulence, and the local two-dimensional waves and their breakdown along with the deformation of the $\Lambda$ shape occur in the transitional boundary layer.

\section{Hot-wire measurement}

For a quantitative investigation of the boundary layer transition due to free stream turbulence, hot-wire measurements are performed in the boundary layer. Figure 10 shows the streamwise changes in the intermittency and energy that were estimated from the streamwise fluctuation according to the relation $E=\left(u_{r m s} / U_{\infty}\right)^{2}$. The wall-normal position is approximately half of the boundary layer thickness, $y / \delta^{*}=1.2$, where $u_{r m s}$ has a peak before the intermittency increases for both cases. $\delta^{*}$ is the displacement thickness of the Blasius boundary 


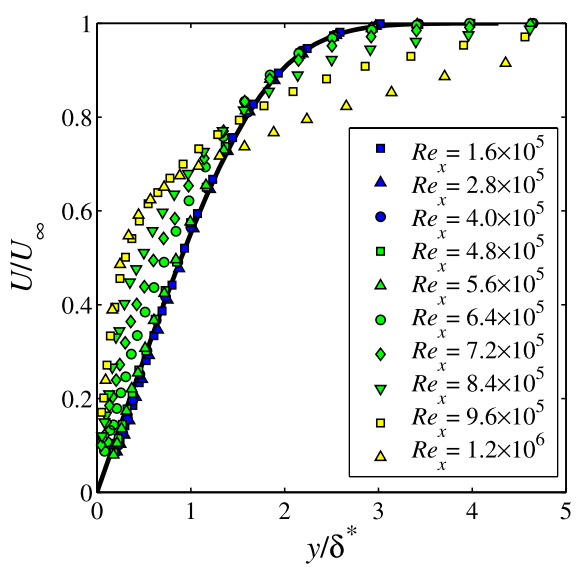

Fig. 11 Mean velocity profiles for grid A.

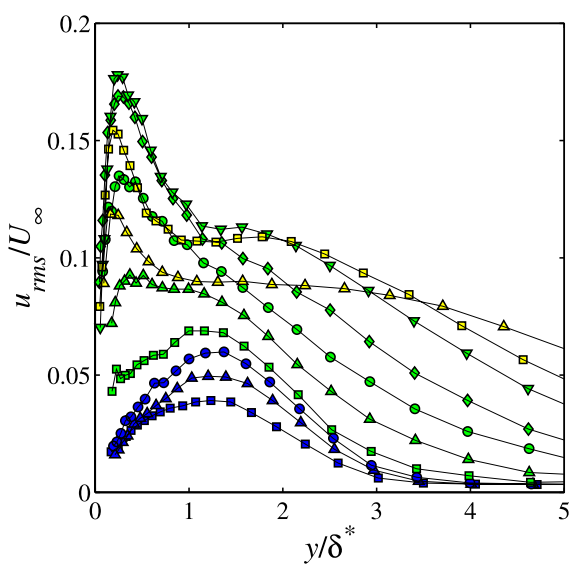

Fig. 12 Velocity fluctuation profiles for grid A. Symbols are the same as those in Fig. 11.

layer. The intermittency is calculated according to the method of Kuan et al. ${ }^{(14)}$, the so-called "dual-slope method." Turbulence durations are detected by a threshold value and the second deviations of the high-pass filtered hot wire signals as a criterion function. The intermittency increases with decrease in the threshold value and the slope folds at a certain point because the signals originally contain more than two components from different characteristic sources. The first slope corresponds to the component of turbulence; hence, the actual intermittency is estimated by the extrapolation of the slope to a threshold value of zero. In this method, the threshold value is automatically determined so that value of intermittency can be obtained without employing artificial parameters. $x_{\gamma}$ is the streamwise position normalized by one at $\gamma=0.5$ as

$$
x_{\gamma}=\frac{x}{x_{\gamma=0.5}}=\frac{R e_{x}}{R e_{x, \gamma=0.5}},
$$

where $R e_{x, \gamma=0.5}=6.89 \times 10^{5}$ for grid A and $R e_{x, \gamma=0.5}=2.84 \times 10^{5}$ for grid $\mathrm{B}$. With this normalization, the intermittency curves in both cases are generally similar in terms of an increase from zero at $x_{\gamma}=0.5$ and saturation at the value of one after $x_{\gamma}=1.3$. It should be mentioned that the boundary layer properties are never disturbed by the spot generation for $R e_{x}<3.2 \times 10^{5}$ in the case of grid A and for $\operatorname{Re}_{x}<1.2 \times 10^{5}$ in the case of grid B because of zero intermittency in this region during the measurements. The streamwise position at $x_{\gamma}=1$ agrees with that at which turbulent spots are observed in the flow visualization. The disturbance energy in the case of grid A increases in proportion to the streamwise distance until the intermittency rises, and subsequently the slope of the energy growth curve suddenly shows a sharp increase. After reaching the peak value, it asymptotically approaches the turbulence value. In the case of grid $\mathrm{B}$, the variation of the disturbance energy is similar to that in the case of grid A, except indistinctness of the slope increase with the intermittency rise. The linear growth of energy at the middle of the boundary layer before the generation of turbulent spots is in agreement with the nonmodal theory ${ }^{(8)(16)}$. The shape factor that is given by $H=\delta^{*} / \theta$, where $\theta$ is the momentum thickness of the boundary layer, decreases with an increase in the intermittency in both cases. It should be noted that the shape factor in the case of grid B starts to decrease before the intermittency begins to increase. This is because of the momentum transfer caused by the streaky structures. This phenomenon was also observed by Westin et al. ${ }^{(13)}$ and Matsubara et al. ${ }^{(9)}$

Figures 11 and 12 show the mean and fluctuation profiles of the streamwise velocity for grid A. The mean velocity profiles up to $R e_{x}=4.0 \times 10^{5}$ are in good agreement with the Blasius profile. There is no systematic tendency in the deviation of the mean velocity from the Blasius profile with a change in the streamwise position up to $R e_{x}=5.6 \times 10^{5}$. The mean velocity downstream accelerates close to the wall and decelerates in the upper part of the 


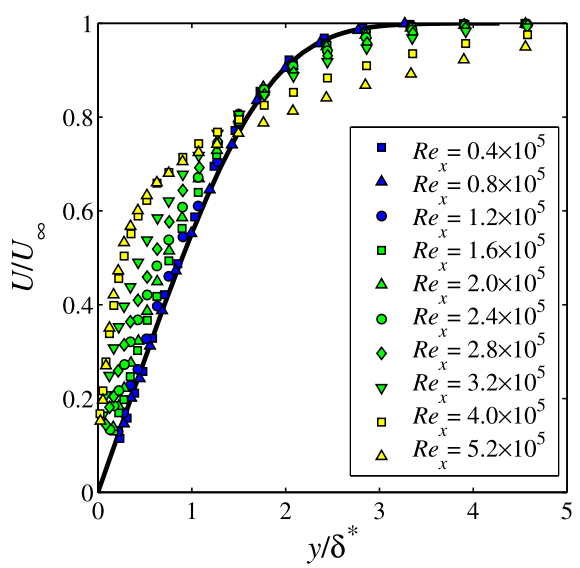

Fig. 13 Mean velocity profiles for grid B.

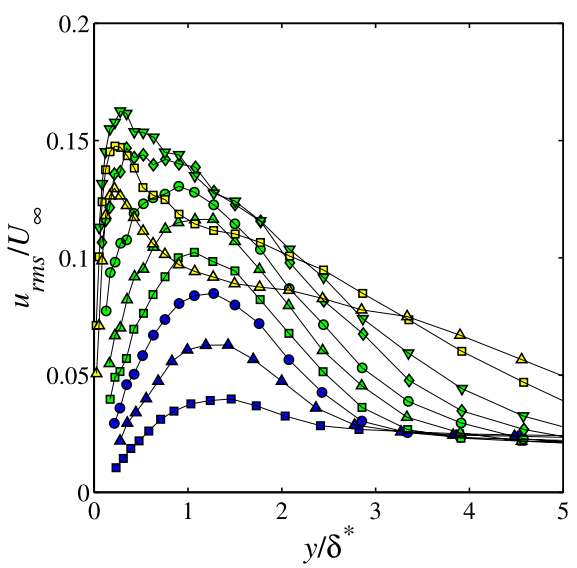

Fig. 14 Velocity fluctuation profiles for grid B. Symbols are the same as those in Fig. 13.

boundary layer; subsequently, it corresponds to a typical turbulent profile at $\operatorname{Re}_{x}=1.2 \times 10^{6}$. This is due to an increase in the intermittency. In Fig. 12, the profile of $u_{r m s}$ has a peak at the middle of the boundary layer, particularly at $R e_{x}=4.0 \times 10^{5}$, and the value of $u_{r m s}$ gradually increases with the streamwise position. In the velocity fluctuation profile for $R e_{x}=4.8 \times 10^{5}$, a rapid increase is observed close to the wall. The intermittency begins to increase at this position, as shown in Fig. 10, and the emergence of two-dimensional waves and $\Lambda$ shape structures are often observed in the visualization in the range around $R e_{x}=4.8 \times 10^{5}$. It cannot be judged whether the fluctuation increase near the wall is due to the generation of turbulent spots or another kind of disturbance such as the local two-dimensional waves observed in the flow visualization of Fig. 6.

Figure 13 shows the streamwise velocity profiles in the case of grid B. The profiles up to $R e_{x}=0.8 \times 10^{5}$ are in good agreement with the Blasius profile. The profiles start to deviate from the Blasius profile at $R e_{x}=1.2 \times 10^{5}$ as the velocity accelerates near the wall and decelerates in the upper region of the boundary layer. This deviation causes a reduction in the shape factor, which was mentioned before. This deviation is not due to turbulent spot generation since the intermittency is still zero at these streamwise positions. Westin et al. ${ }^{(13)}$ and Matsubara et al. ${ }^{(9)}$ also reported this phenomenon, and Westin et al. suggested that the deviation is caused by an increase in the Reynolds stress due to a disturbance in the boundary layer. Downstream of $R e_{x}=1.6 \times 10^{5}$, the profile gradually changes to a turbulence profile with an increase in the intermittency; the profile then becomes a typical turbulent profile at $R e_{x}=5.2 \times 10^{5}$. In Fig. $14, R e_{x}=5.2 \times 10^{5}$, the fluctuation profiles have a peak at the middle of the boundary layer up to $R e_{x}=2.0 \times 10^{5}$, which is similar to the case of grid A. However, the peak grows by over $10 \%$ and then the peak position shifts close to the wall and downstream. In the case of grid B, the peak growth near the wall that was observed in the case of grid A did not exist. This change in the fluctuation coincides with the results of Westin et al. ${ }^{(13)}$ and Matsubara et al. ${ }^{(9)}$

Figure 15 shows a comparison of the profiles of the velocity fluctuation with the predicted profiles of the nonmodal growth theory ${ }^{(8)}$. The profiles normalized with the peak values are self-similar over most of the boundary layer and fit the theoretical profiles, except for the regions around a boundary layer edge and a free stream where influence of the free stream turbulence exists. This comparison does not strongly indicate that the disturbances in the boundary layers are due to nonmodal growth because the profiles can be explained by considering other disturbances such as the Stewartson mode ${ }^{(17)}$.

The spectra of the streamwise fluctuations at $y / \delta^{*}=0.7$ in the case of grid A are shown in Fig. 16. This wall-normal position is approximately the peak position of the T-S disturbance predicted by the parallel linear theory. The vertical axis is multiplied by the horizontal axis 


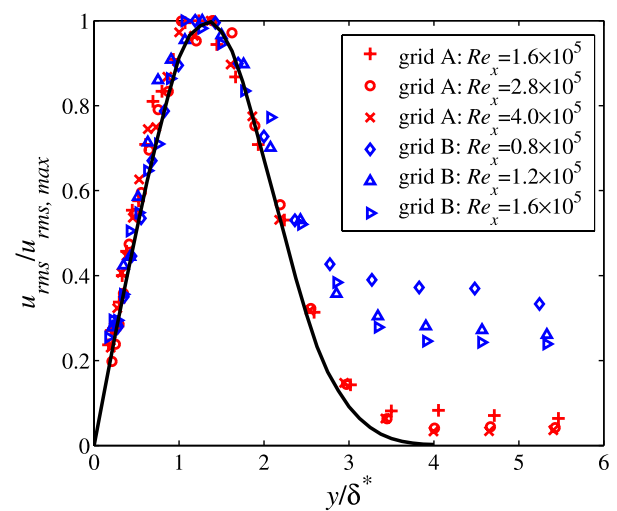

Fig. 15 Comparison with the predictions of nonmodal growth theory ${ }^{(8)}$.

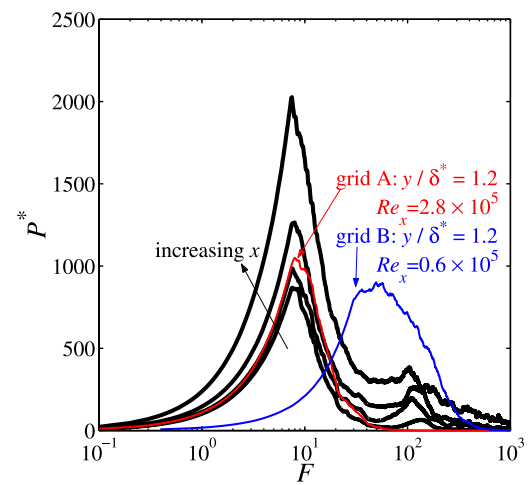

Fig. 16 Energy density spectra at different streamwise positions starting at $R e_{x}=4.0 \times$ $10^{5}$ and at intervals of $0.4 \times 10^{5} . P^{*}=F \times$ power.

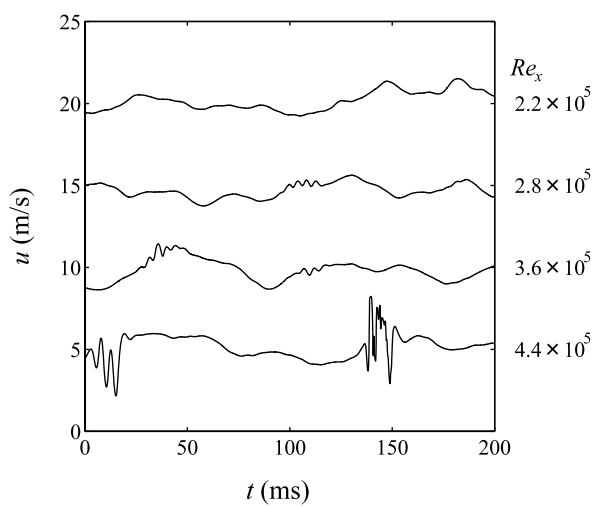

Fig. 17 Velocity signals from different streamwise position at $y / \delta^{*}=0.7$. Reference velocity is shifted from 0 to $15 \mathrm{~m} / \mathrm{s}$ with $5 \mathrm{~m} / \mathrm{s}$ space.

value so that the area represents the energy. The energy increases downstream at around $70<F<200$ as well as around $F=7$. The frequencies of the weak waves determined from the visualization are around $F=130$; they are calculated by using the wavelength and phase velocity measured from sequences such as the sequence shown in Fig. 7. A comparison among the spectra at the maximum fluctuation is also performed in Fig. 16. The peak frequency in the case of grid A is one order lower than that in the case of grid B. This peak frequency is of the order of $1 \mathrm{~m}$, which is too large as compared to the typical streamwise length of streaky structures.

Selected hot-wire traces at $y / \delta^{*}=0.7$ in the case of grid A are shown in Fig. 17. Each signal is measured at a different time. At $R e_{x}=2.8 \times 10^{5}$ and $R e_{x}=3.6 \times 10^{5}$, oscillations of approximately $200 \mathrm{~Hz}$ appear to be superimposed on low-frequency undulations that have a timescale of the order of $100 \mathrm{~ms}$; these undulations are also observed at $R e_{x}=2.2 \times 10^{5}$. The normalized frequency of these oscillations is approximately $F=130$, which corresponds to the peak of the spectra shown in Fig. 16. The number of waves in each packet ranges from three to five; a similar number of waves were also observed in the wave packets observed in the visualization. Around $t=150 \mathrm{~ms}$ at $R e_{x}=4.4 \times 10^{5}$, the very large amplitude waves and high-frequency waves are attributed to the turbulence generation. This streamwise position corresponds to the position where the intermittency begins to rise, $x_{\gamma}=0.64$.

The following analyses is performed in order to determine whether the wave packets observed in both flow visualization and hot-wire measurements are related to T-S waves. Figures 18 and 19 show profiles of the velocity fluctuation after low-pass and band-pass filtering. The cutoff frequency for the low-pass filter is $F=10$ and the band-pass frequencies correspond to $F=130 \pm 20$. From a comparison with the unfiltered profiles shown in Fig. 12, it is clear 


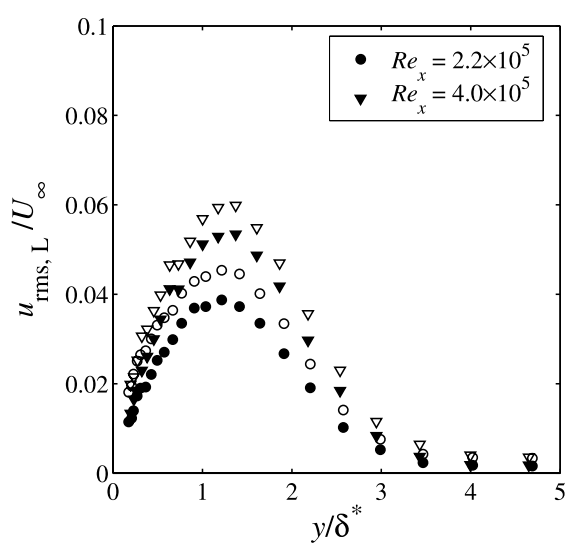

Fig. 18 Low-pass filtered disturbance profiles. The open symbols are original profiles and the solid symbols are fitted profiles.

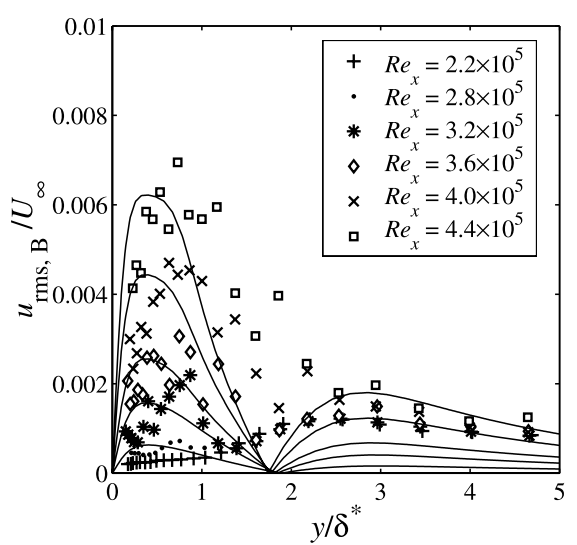

Fig. 19 Band-pass filtered disturbance profiles. The theoretical line for $R e_{x}=2.2 \times 10^{5}$ is neglected.

that the low-pass filtered fluctuations in Fig. 18 dominate the entire energy range. The profiles in Fig. 19 indicate properties that are entirely different from those of the low-pass filtered fluctuations. The fluctuations are considerably small and grow downstream with peaks around $y / \delta^{*}=0.7$. Their profiles resemble the profile of the absolute values of the T-S wave amplitudes (shown as solid lines), which are calculated by using the linear stability theory with a parallel flow assumption with $F=130$. The scattering of the data is due to the significantly short duration of the T-S waves as compared to the integral duration. Exponential growth is not evident in Fig. 19. In Kendall' s experiments ${ }^{(18)}$, the pressure fluctuation do not follow exponential growth depending on the free stream turbulence intensity.

Figure 20 shows the neutral curve of the T-S wave growth and compares the appearance of the wave packets in the flow visualization with those derived from the velocity signals. The frequency and Reynolds number of the two-dimensional wave packets are estimated by using the wavelength and phase velocity of the waves determined from the video sequences. The two-dimensional waves from the velocity signals were detected visually. Good coincidence between the waves obtained from the flow visualization and those derived from the velocity signals with regard to the Reynolds numbers and frequencies of their appearance proves that the wavy motion in the flow visualization and the signal oscillations are identical, though it is difficult to distinguish whether they are two-dimensionally or three-dimensionally deformed relative to the velocity signals. All the plots are in the neighborhood of the upper branch of the neutral curve and some of them are outside the unstable region. However, it is thought that the wave packets were in an unstable state before they appeared. In Kendall' s results ${ }^{(18)}$, the frequencies of these waves were higher than those of the T-S instabilities with the highest amplification. Furthermore, as observed in Fig. 21, the phase velocity $c_{r, \text { m }}$ estimated from the video sequence of the flow visualization agrees well with the theoretically predicted phase velocity $c_{r \text {, p }}$ that used the wavelength and Reynolds number obtained from the flow visualization.

To conclude, these results indicate that the disturbances triggering the transition in the boundary layer subjected to a relatively weak free stream turbulence are wave packets of locally generated T-S waves. These T-S wave packets are quickly deformed to the $\Lambda$ shape and break down to turbulence. It is also confirmed that the transition process is drastically altered by the property of free stream turbulence. In the case of grid B, for which streaky structures are observed in the flow visualization, the transition process and the character of the triggering disturbance are in good agreement with the results of the experiment of Matsubara et al. ${ }^{(9)}$ These authors also investigated the transition caused by the streaky structures at a free stream intensity of around $1.2 \%$. This value is not very far from the $v_{r m s, \infty} / U_{\infty}$ value of $0.77 \%$ in the case of grid A. It is assumed that the drastic alteration of the transition process is 


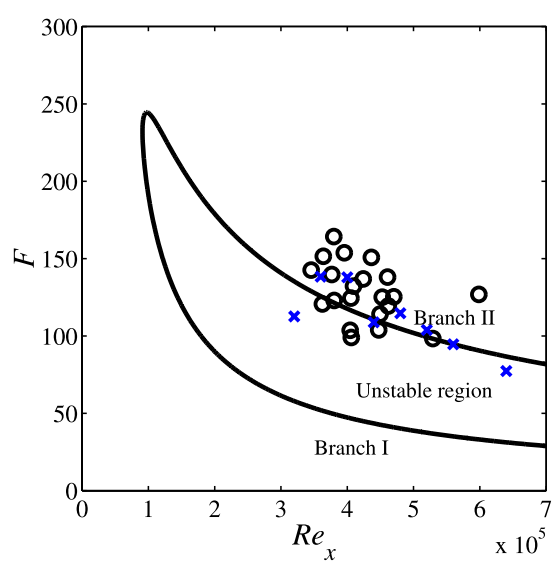

Fig. 20 The neutral stability curve and the normalised disturbance frequencies. ०: wave packets observed from flow visualization. $\times$ : spectral peaks from velocity signals.

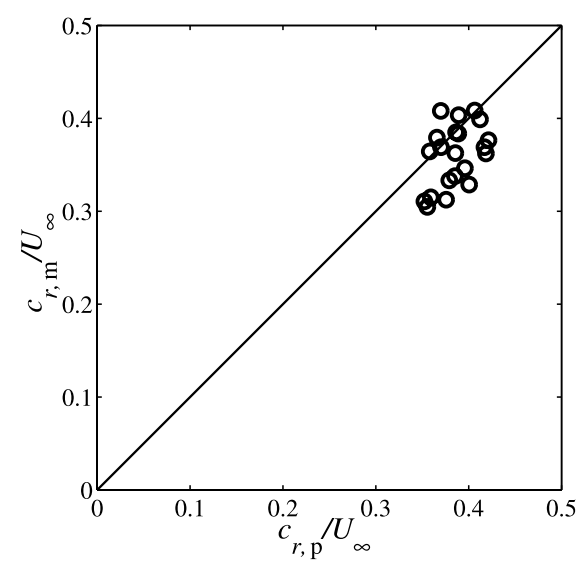

Fig. 21 Comparison of experimental and theoretical phase velocities.

due to not only the intensity of the free stream turbulence but also the variety of their spectra.

In the case of grid $\mathrm{A}$, in the flow visualization, large-scale structures in both the spanwise and streamwise directions exist at this streamwise position and there are no distinguishable streaky structures. This case differs from that of grid B in that the shape factor shows no tendency to decrease before an increase in the intermittency occurs, even though the lowfrequency disturbance grows. From the flow visualization, it seems that this low-frequency disturbance does not affect the transition process. The peak frequency of this disturbance observed in the velocity spectra corresponds to an order similar to the length of the test plate, and it is much lower than when streaky structures exist. It appears that we need more detailed data, particularly on the spanwise correlation of velocities, in order to understand this lowfrequency disturbance and its role in the transition.

\section{Conclusion}

For the elucidation of the process of transition from a laminar to a turbulence in a boundary layer subjected to a low-intensity free stream turbulence, flow visualization and hot-wire measurements were conducted. The flow visualization clearly revealed a transition scenario in which a local Tollmien-Schlichting wave packet appears and forms $\Lambda$ shape structures before breaking down to turbulence and generating a turbulent spot. Quantitative measurements by using a hot-wire anemometer also confirmed the existence of local T-S waves in terms of its frequency, phase velocity and wall-normal distribution and its breakdown to turbulence with generating a turbulent spot.

The boundary layer transition due to a low-intensity free stream turbulence is triggered by the breakdown of the wave packets, which is in contrast to the streak breakdown that occurs in the case of high free stream turbulence. Further research is needed for studying the threshold between two transition paths; these studies should consider both the directional spectral components of the free stream turbulence.

\section{References}

( 1 ) Schubauer, G. B. and Skramstad, H.K., Laminar Boundary-Layer Oscillations and Transition on a Flat Plate, NACA Technical Report, 909, (1948)

( 2 ) Klebanoff, P. S., Effect of Free Stream Turbulence on the Laminar Boundary Layer, Bulletin of American Physical Society, Vol. 10, No. 11, (1971), pp. 1323.

( 3 ) Kendall, J. M., Experiments on Boundary-Layer Receptivity to Freestream Turbulence, AIAA paper 98-0530, (1998) 
( 4 ) Kendall, J. M., Experimental Study of Disturbances Produced in a Pre-Transitional Laminar Boundary Layer by Week Freestream Turbulence, AIAA paper 85-1695, (1985)

( 5 ) Gaster, M. and Grant, I., An Experimental Investigation of the Formation and Development of a Wave Packet in a Laminar Boundary Layer, Proceedings of the Royal Society of London. Series A, Vol. 347, (1975), pp. 253-269.

( 6 ) Arnal, D. and Juillen, J. C., Contribution Experimentale a L'etude de la Receptivite D'une Couche Limite Laminaire, a la Turbulence de L'ecoulement General, Rapport Technique, 1/5018, (1978), ONERA.

( 7 ) Kosorygin, V. S. and Polyakov, N. Ph., Laminar Boundary Layers in Turbulent Flows, In Laminar-Turbulent Transition (ed. D. Arnal \& R. Michel), (1990), pp. 573-578, Springer.

( 8 ) Luchini, P., Reynolds-Number-Independent Instability of the Boundary Layer Over a Flat Surface: Optimal Perturbations, Journal of Fluid Mechanics, Vol. 404, (2000), pp. 289-309.

( 9 ) Matsubara, M. and Alfredsson, P. H., Disturbance Growth in Boundary Layers Subjected to Free-Stream Turbulence, Journal of Fluid Mechanics, Vol. 430, (2001), pp. 149-168.

(10) Klingmann, B. G. B., Boiko, A. V., Westin, K. J. A., Kozlov,V. V. and Alfredsson, P. H., Experiments on the Stability of Tollmien-Schlichting Waves, European Journal of Mechanics B-Fluids, Vol. 12, No. 4, (1993), pp. 493-514.

(11) Uberoi, M. S., Equipartition of Energy and Local Isotropy in Turbulent Flows, Journal of Applied Physics, Vol. 28, No. 10 (1957), pp. 1165-1170.

(12) Grant, H. L. and Nisbet, I. C. T., The Inhomogeneity of Grid Turbulence, Journal of Fluid Mechanics, Vol. 2, (1957), pp. 263-272.

(13) Westin, K. J. A., Boiko, A. V., Klingmann, B. G. B., Kozlov, V. V. and Alfredsson, P. H., Experiments in a Boundary Layer Subjected to Free Stream Turbulence. Part 1. Boundary Layer Structure and Receptivity, Journal of Fluid Mechanics, Vol. 281, (1994), pp. 193-218.

(14) Kuan, C. and Wang, T., Some Intermittent Behavior of Transitional Boundary Layer, AIAA paper 89-1890, (1989)

(15) Fransson, J. H. M., Matsubara, M. and Alfredsson, P. H., Transition Induced by FreeStream Turbulence, Journal of Fluid Mechanics, Vol. 527, (2005), pp. 1-25.

(16) Andersson, P., Berggren, M. and Henningson, D. S., Optimal Disturbances and Bypass Transition in Boundary Layers, Physics of Fluids, Vol. 11, No. 1, (1999), pp. 134-150.

(17) Libby, P. A. and Fox, H., Some Perturbation Solutions in Laminar Boundary-Layer theory. Part 1. The momentum equation, Journal of Fluid Mechanics, Vol. 17, (1964), pp. 433-449.

(18) Kendall, J. M., Boundary Layer Receptivity to Freestream Turbulence, AIAA paper 901504, (1990) 